\title{
Using $\mathrm{CT}$ texture analysis to differentiate between peripheral lung cancer and pulmonary inflammatory pseudotumor
}

\author{
Chenlu Liu ${ }^{1,2+}$, Changsheng Ma² ${ }^{2 \dagger}$ Jinghao Duan², Qingtao Qiu², Yanluan Guo ${ }^{3}$, Zhenhua Zhang ${ }^{1}$ and Yong Yin ${ }^{2 *}$
}

\begin{abstract}
Background: This study is to distinguish peripheral lung cancer and pulmonary inflammatory pseudotumor using CT-radiomics features extracted from PET/CT images.

Methods: In this study, the standard 18F-fluorodeoxyglucose positron emission tomography/ computed tomography (18 F-FDG PET/CT) images of 21 patients with pulmonary inflammatory pseudotumor (PIPT) and 21 patients with peripheral lung cancer were retrospectively collected. The dataset was used to extract CT-radiomics features from regions of interest (ROI), The intra-class correlation coefficient (ICC) was used to screen the robust feature from all the radiomic features. Using, then, statistical methods to screen CT-radiomics features, which could distinguish peripheral lung cancer and PIPT. And the ability of radiomics features distinguished peripheral lung cancer and PIPT was estimated by receiver operating characteristic (ROC) curve and compared by the Delong test.

Results: A total of 435 radiomics features were extracted, of which 361 features showed relatively good repeatability (ICC $\geq 0.6$ ). 20 features showed the ability to distinguish peripheral lung cancer from PIPT. these features were seen in 14 of 330 Gray-Level Co-occurrence Matrix features, 1 of 49 Intensity Histogram features, 5 of 18 Shape features. The area under the curves (AUC) of these features were $0.731 \pm 0.075,0.717,0.748 \pm 0.038$, respectively. The $P$ values of statistical differences among ROC were 0.0499 (F9, F20), 0.0472 (F10, F11) and 0.0145 (F11, Mean4). The discrimination ability of forming new features (Parent Features) after averaging the features extracted at different angles and distances was moderate compared to the previous features (Child features).
\end{abstract}

Conclusion: Radiomics features extracted from non-contrast CT based on PET/CT images can help distinguish peripheral lung cancer and PIPT.

Keywords: Radiomics features, Peripheral lung cancer, PET/CT, Pulmonary inflammatory pseudotumor

\footnotetext{
*Correspondence: yinyongsd@126.com

${ }^{\dagger}$ Chenlu Liu and Changsheng Ma contributed equally to this work.

${ }^{2}$ Department of Radiotherapy, Shandong Cancer Hospital and Institute,

Shandong First Medical University and Shandong Academy of Medical

Sciences, Jinan 250117, Shandong Province, China

Full list of author information is available at the end of the article
}

(c) The Author(s). 2020 Open Access This article is licensed under a Creative Commons Attribution 4.0 International License, which permits use, sharing, adaptation, distribution and reproduction in any medium or format, as long as you give appropriate credit to the original author(s) and the source, provide a link to the Creative Commons licence, and indicate if changes were made. The images or other third party material in this article are included in the article's Creative Commons licence, unless indicated otherwise in a credit line to the material. If material is not included in the article's Creative Commons licence and your intended use is not permitted by statutory regulation or exceeds the permitted use, you will need to obtain permission directly from the copyright holder. To view a copy of this licence, visit http://creativecommons.org/licenses/by/4.0/ The Creative Commons Public Domain Dedication waiver (http://creativecommons.org/publicdomain/zero/1.0/) applies to the data made available in this article, unless otherwise stated in a credit line to the data. 


\section{Background}

Lung cancer is the world's leading cause of cancerrelated deaths and a highly malignant tumor $[1,2]$, According to the location of the tumor, lung cancer can be divided into peripheral lung cancer and central lung cancer, and more than $70 \%$ of all lung cancers are peripheral lung cancer [3]. lung cancer can be divided into adenocarcinoma, squamous cell carcinoma, small cell lung cancer and large cell lung cancer depending on the histological and cytological types of tumors. In recent years, with the improvement of the level of cancer diagnosis and treatment [4], the mortality rate of lung cancer has been on a downward trend, but lung cancer is still the leading cause of death [5]. About $70 \%$ of the patients with lung cancer are in the advanced stage at the time of initial diagnosis [6], and even some patients have already had metastasis, and if patients with lung cancer can be accurately diagnosed and undergo early surgery, the 10year survival rate can reach $88 \%$ [7]. thus, the early diagnosis and timely treatment of patients with lung cancer are very important [8].

The good diagnostic effect of computed tomography (CT) has been achieved in the diagnosis of lung cancer. However, the traditional CT diagnosis of lung lesions is mainly based on morphology, which has certain limitations in the differential diagnosis of benign and malignant tumors $[9,10]$.

In recent years, 18F-fluorodeoxyglucose positron emission tomography/computed tomography (18F-FDG $\mathrm{PET} / \mathrm{CT}$ ) has achieved a great effect in the examination of chest lesions [11], and has been widely used in clinic [8]. Some reports have shown that PET/CT is more effective than conventional $\mathrm{CT}$ in identifying benign and malignant chest lesions [12,13]. However, FDG is not a drug specifically ingested by cancer [13]. In most cases, the cumulative increase in metabolic activity of inflammatory cells will lead to an increase in FDG uptake, which is false positive on PET-CT. To some extent, these lesions can also mimic the biological behavior of malignant tumors. Even if experienced nuclear medicine physicians manage to distinguish between benign and malignant FDG uptake, The result is still far from satisfactory. This is a great challenge for clinicians and nuclear medicine doctors, such as the differentiation of pulmonary inflammatory pseudotumor (PIPT) and peripheral lung cancer [14, 15].

The CT signs of PIPT are usually complex, variable and unspecific, and showing round or oval, well-defined solitary peripheral nodules or masses [16-19], accounting for about $1 \%$ of adult lung tumors [20]. It is a rare disease characterized by pulmonary neoplastic hyperplasia of inflammatory cells, which is very similar to malignant tumors of the lung [21, 22]. PIPT occasionally has a process of invasive proliferation and usually shows false positive on PET/CT [18]. Due to the overlap between PIPT and peripheral lung cancer in histopathology, biological behavior, clinical manifestations and imaging features [20, 23], the clear diagnosis and differentiation often can't be made by clinicians and nuclear medicine physicians.

Radiomics widely used in modern medical research, due to radiomics can quantitative tumor heterogeneity with the advantages of repeatable, non-invasive, free from time and space constraints and low-cost [24, 25]. In recent years, radiomics plays a more and more important role in the qualitative diagnosis of tumors, the differentiation of benign from malignant tumors and the evaluation of prognosis of radiotherapy response. The purpose of this study is to explore whether PIPT and peripheral lung cancer can be distinguished by radiological analysis.

\section{Methods \\ Radiomics workflow}

The raidomics flow of this study included: (1) images acquisition, (2) image segmentation, (3) feasure extraction, (4) data analysis. All the steps are shown in Fig. 1.

\section{Patients}

This study was approved by the Ethics Review Committee of Shandong Cancer Hospital and informed consent was waived. The collection of patient materials in this study was carried out in two steps. The first step enrolled PIPT patients who had undergone 18F-FDG PET/ CT scans in our hospital from May 2015 to October 2019, and their pathological diagnosis was available. In the second step, the images of the patients with peripheral lung cancer were included randomly. The criteria for patient inclusion were as follows: (I) the patient underwent PET/CT examination for the first time; (II) the patient had no history of diabetes; (III) the maximum diameter of the lesion was not less than $1.5 \mathrm{~cm}$; (IV) the patient had complete clinical materials and pathological reports; (V) standardized uptake value of lesions $\geq 2.5$.

\section{Patient images acquisition}

The 18F-FDG PET/CT images of all patients were obtained by the same hybrid PEC/CT scanner (Philips Healthcare, Cleveland, $\mathrm{OH}$ ). The patient fasted for more than $6 \mathrm{~h}$, and the blood glucose level of all patients was normal before the scan. PET/CT scans were completed $60 \mathrm{~min}$ after intravenous injection of 18F-FDG at 4.4 $\mathrm{MBq} / \mathrm{kg}$. After attenuation correction and iterative reconstruction, the PET images were used for multi-plane and multi-image imaging, and the images were fused with the $\mathrm{CT}$ images. The initial $\mathrm{CT}$ image acquisition was conducted with slice thickness $2 \mathrm{~mm}$ (120KVp, 206 


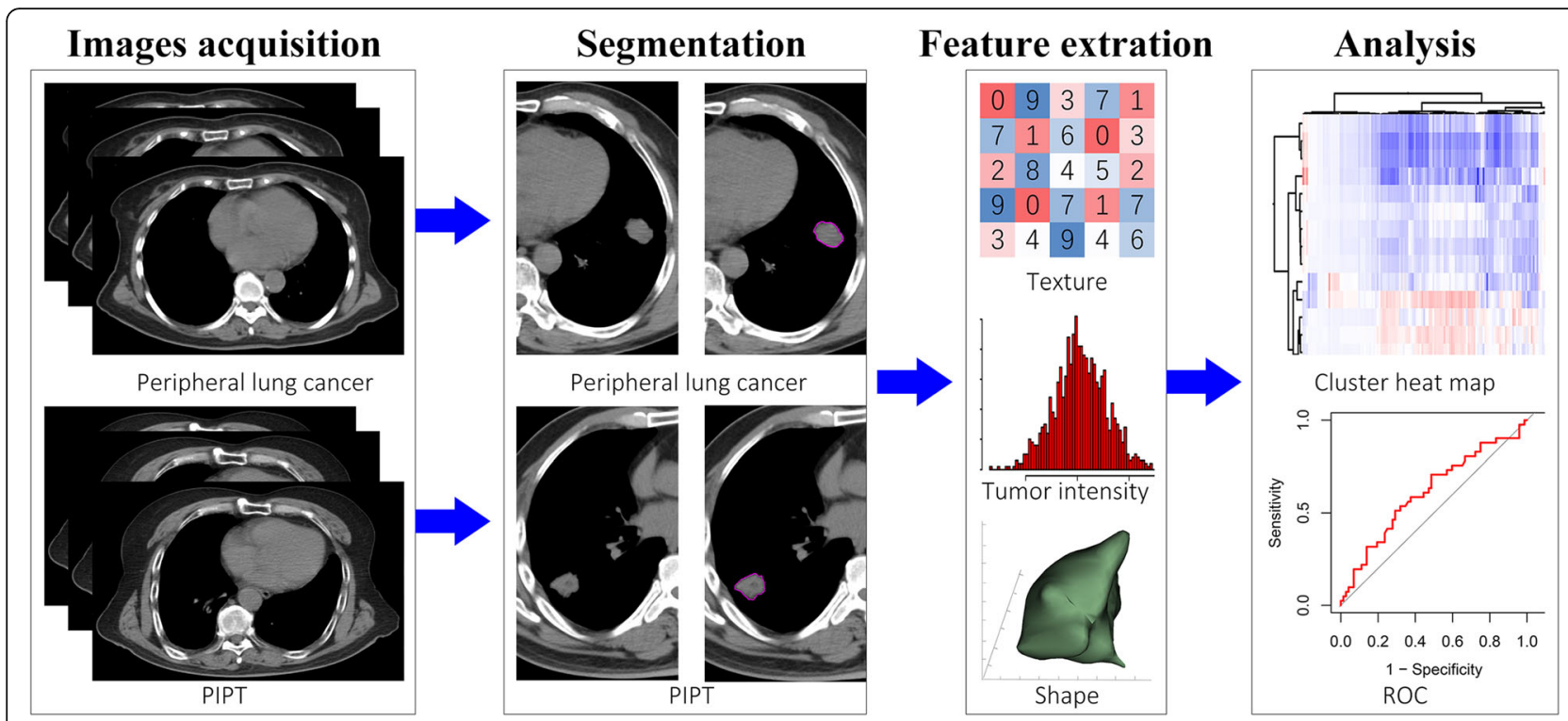

Fig. 1 The workflow of this study

$\mathrm{mA}$ ), and reconstructed to a $512 \times 512$ matrix (voxels size, $1.17 \times 1.17 \times 5 \mathrm{~mm}^{3}$ ). Then, the obtained image data can be viewed in coronal, sagittal, and axial planes using image workstation.

\section{Region of interests (ROI) segmentation}

The total process of image segmentation was completed by three experienced doctors. All the image data were imported into the MIM software (Cleveland, $\mathrm{OH}$ ), and the total process of image segmentation was completed by three experienced radiologists. In the first group of experiments, ROI was manually delineated by a radiologist with 15 years of experience on the MIM software and supervised by another nuclear medicine physician with 20 years of experience, a clinical chest doctor with 20 years of experience to examine and correct all the delineated images. In the second group of experiments, ROI was manually delineated by a nuclear medicine physician with 20 years of experience, The other two doctors checked all images and discussed whether to make changes. While the three senior doctors were working, they were unable to access all patient-related medical information. Finally, all the images of the two groups of experiments were exported by a doctor.

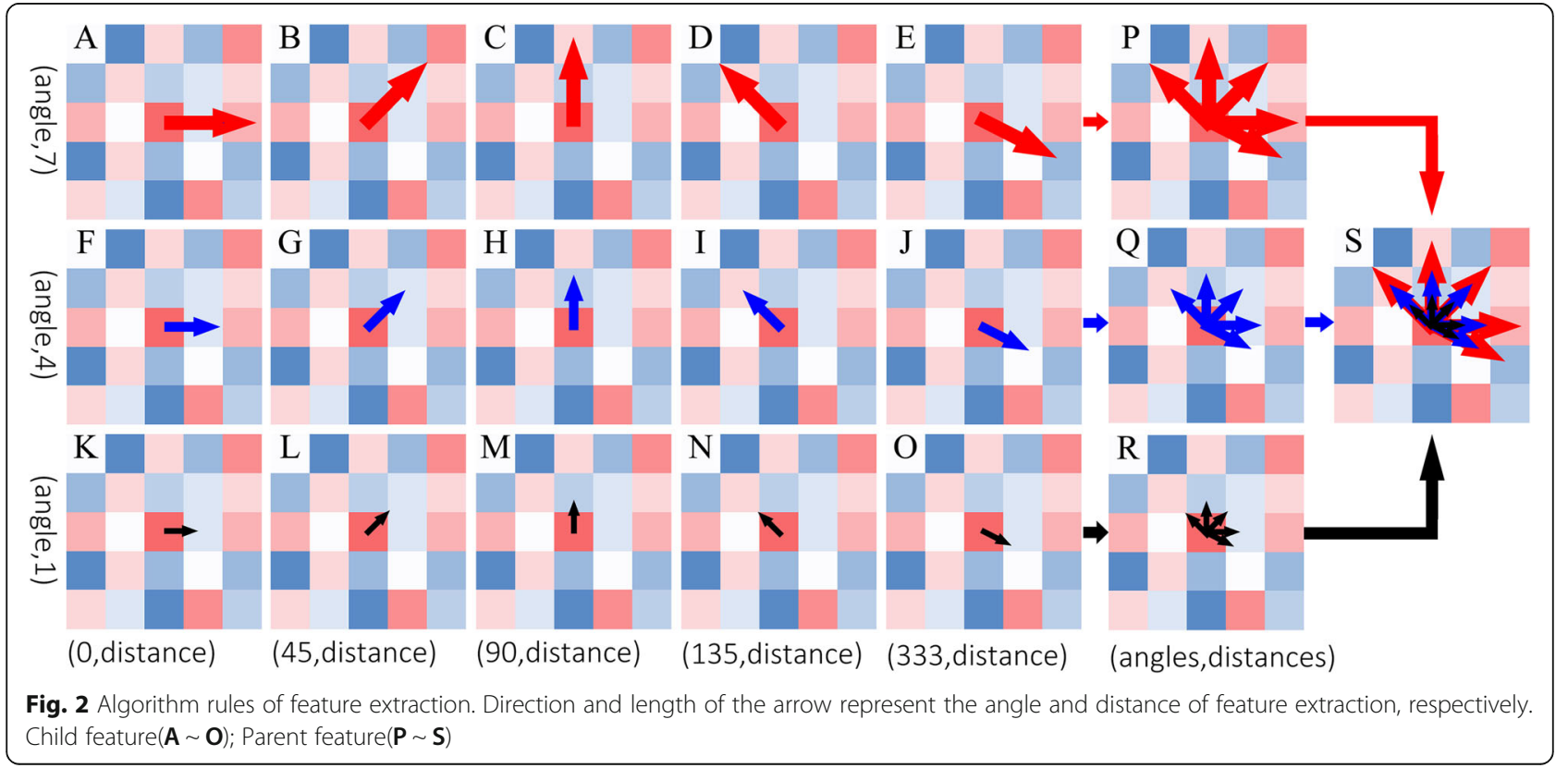




\section{Extraction of radiomic features}

All radiomics feature were automatically using imaging Biomarker Explorer (IBEX) software (MD Anderson Cancer Center, TX, USA), which is an open infrastructure software platform that flexibly supports common radiomics workflow tasks such as multimodality image data import and review, development of feature extraction algorithms, model validation, and consistent data sharing among multiple institutions [26]. In this study, we named the features with different names under the same matrix as parent features, and the features with the same name extracted from different angles and different distances as child features, in other words, we did not average the feature values of the same name from the same matrix extracted from different angles and distances.

This process had been shown in Fig. 2 .

\section{Statistical analysis}

The Intra-class correlation coefficient (ICC) was used to evaluate reproducibility [27], ICC ranges from 0 to 1 , where 0 indicates no repeatability and 1 indicates full repeatability. Intraclass correlation coefficients were defined as poor $(\mathrm{ICC}<0.4)$, fair $(0.4 \leq \mathrm{ICC}<0.6)$, good $(0.6 \leq \mathrm{ICC}<0.75)$, and excellent $(\mathrm{ICC} \geq 0.75)$ as reported previously [28]. Only the feature with excellent repeatability will be included in the follow-up analysis. The Mann-Whitney U test [29] was performed to analyze the differences between PIPT and peripheral lung cancer, this process of statistical analysis was implemented on Matlab (version 2014b, www.mathworks.com). Radiomics features that can distinguish between peripheral lung cancer and PIPT were obtained using logistic regression analysis on Medcalc (version 18.2.1, http://www.medcalc.org). The diagnostic performance of the radiomic features was illustrated by the receiver operating characteristic (ROC) curve with indices of the area under the curve (AUC), confidence Interval, sensitivity and specificity, and compared by the Delong test. The criterion value distinguished peripheral lung cancer and PIPT was determined by the maximum of the Youden index [30] (calculating the sum of specificity and sensitivity at all possible thresholds, then subtracting one), $P$-value was corrected by false discovery rate (FDR) to adjust for multiple comparisons [31], $P$ value $<0.05$ was considered statistically significant. In addition, we average the feature values of the same name extracted from different angles and distances as new features and observe their diagnostic capabilities.

The data from this study were $\mathrm{z}$-score transformed across each radiomic features and displayed as a heat map with ward. D agglomeration method. Figure 3 represents a heat map of all radiomic feature extracted.

\section{Results \\ Patient characteristics}

A total of 21 PIPT patients were enrolled in this study, including 15 males and 6 females (maximum age 74

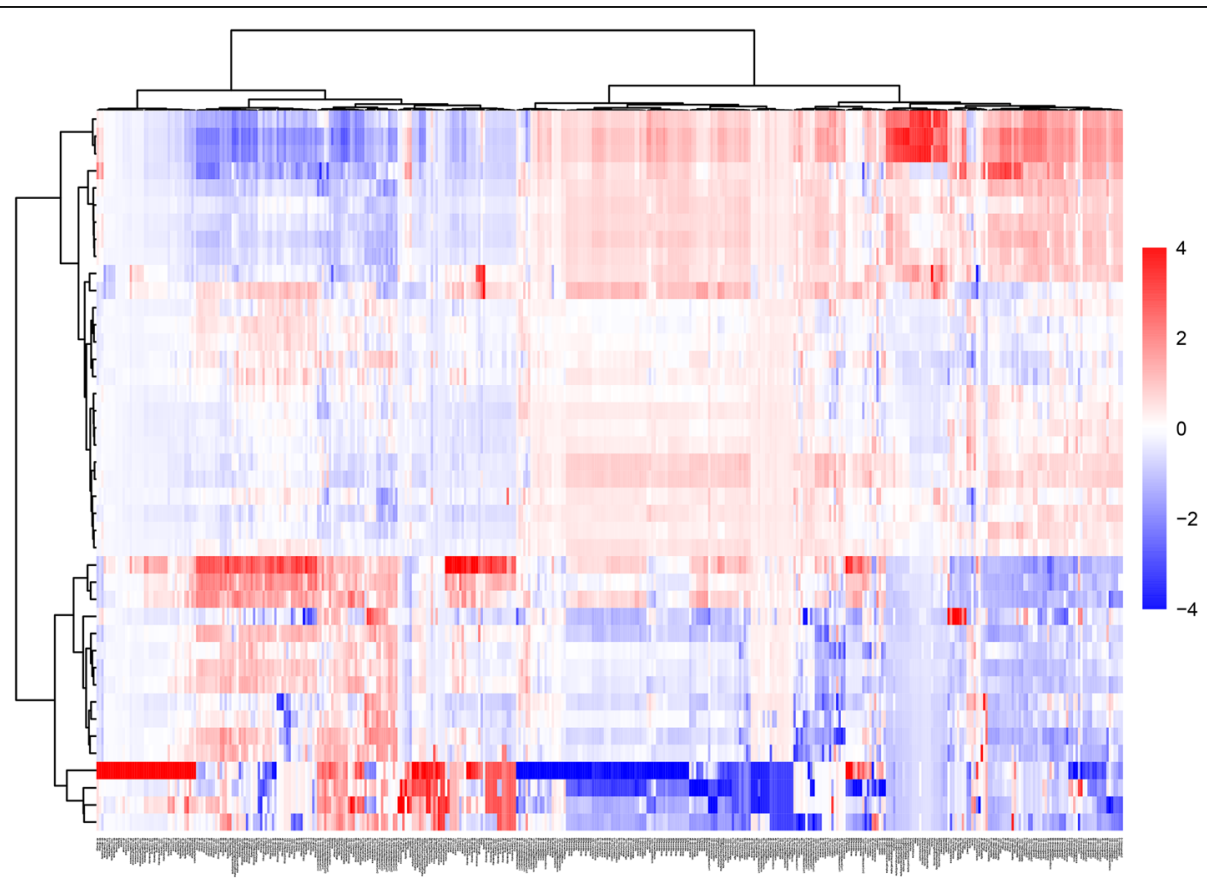

Fig. 3 Unsupervised hierarchical clustering heat map of radiomic features extracted from CT images from 42 patients. In the heat map, each row of the heat map represents a patient, and each column represents a radiomic feature extracted from the patient's $C T$ images. The differences in feature values were visualized using red to represent higher than average and blue to represent lower than average 
Table 1 Clinical case information of patients with peripheral lung cancer and PIPT enrolled in this study

\begin{tabular}{lll}
\hline Characteristics & peripheral lung cancer & PIPT \\
\hline $\begin{array}{l}\text { Age, Mean } \pm \text { SD (years) } \\
\text { Gender }\end{array}$ & $63.62 \pm 8.62$ & $57.68 \pm 8.61$ \\
$\quad$ Male & 15 & 13 \\
$\quad$ Female & 6 & 8 \\
Tumor volume $\left(\mathrm{cm}^{3}\right)$ & & 1.05 \\
$\quad$ Minimum value & 1.65 & 82.08 \\
Maximum value & 64.29 & 18.17 \\
Mean value & 14.62 & \\
Maximum diameter of tumor $(\mathrm{cm})$ & 1.69 \\
Minimum value & 1.61 & 4.97 \\
Maximum value & 6.4 & 2.75 \\
Mean value & 3.30 & \\
SUV & & 2.6 \\
Minimum value & 3.6 & 17.3 \\
Maximum value & 26.2 & 10.46 \\
Mean value & 12.73 & \\
\hline & & \\
\hline
\end{tabular}

years, minimum age 44 years, median age 58 years). In the second step, 21 patients with peripheral lung cancer were randomly collected, including 13 males and 8 females (maximum age 78 years, minimum age 44 years, median age 67 years). 14 were diagnosed with adenocarcinoma, 6 with squamous cell carcinoma, and 1 with atypical carcinoid. The clinical characteristics of the patients in this study were shown in Table 1.

\section{Feature results}

In this study, a total of 435 radiomics features were extracted (composed of 368 texture features, 18 shape features, and 49 tumor intensity features), According to the different calculation methods, these features were divided into 5 categories. Among them, the gray-level cooccurrence matrix (GLCM,22 parent features, 330 child features), gray-level run length matrix (GLRLM, 11 parent features, 33 child features), intensity histogram ( $\mathrm{IH}$, 9 parent features, 49 child features), gray-level neighbor intensity difference matrix (GLNIDM, 5 parent features, none), shape (null,18 patient features, none).

\section{Statistical results}

As shown in Table 2, a total of 361 radiomic features (ICC $\geq 0.75$ ) show good repeatability. Among them, the ability of repeatable shape features is up to $100 \%$ (18/ 18). followed by the features belonging to IH and GLCM reached $85.71 \%$ (42/49), 84.24\% (278/330), respectively.

All statistical differences of PIPT and peripheral lung cancer were tested by the Mann-Whitney U test. A total of 29 feature differences were found to be statistically significant, of which the GLCM has 22 child features, which belong to 5 parent features, respectively, 1 parent feature in IH, 1 parent features in NIDM, 5 parent features in shape, and their child features are all 0 . The 30 radiomics features with statistically significant differences are shown in Table 3.

Binary logistic regression model analysis showed that 23 of the 29 child features were significantly different and could be used to distinguish PIPT from peripheral lung cancer. These 23 child features respectively belong to GLCM (parent feature correlation $(n=8)$, parent feature information measure corr1 $(n=4)$ and parent feature information measure corr2 $(n=4)) 、 \mathrm{IH}$ (parent feature range $(n=0))$ 、 GLNIDM (parent feature texture strength $(n=0)$ ) and shape (parent feature compactness2 $(n=0)$, Roundness $(n=0)$, parent feature spherical disproportion $(n=0)$, parent feature sphericity $(n=0)$ and parent feature surface area $\operatorname{density}(n=0)$ ), respectively. (Table 3).

ROC curves of 25 features were performed to evaluate the ability of features differentiating peripheral lung cancer from PIPT. The curves $(\mathrm{AUC}<0.7)$ was abandoned in this study, because of its limited discriminant ability. Finally, A total of 20 ROC curves of features were obtained in this study. In addition, we calculated the average value of the features at the same angle and different distance and drew ROC curves, which were curve Mean1 and Mean2. we also calculated the average value of the features at different angles and different distances and the drew of ROC curves were curve mean3, mean4, mean5, respectively. The $P$-values of statistical differences among ROC were 0.0499 (F9, F20), 0.0472 (F10, F11), and 0.0145 (F11, Mean4), and the others were $0.5908 \pm 0.2803$. All ROC curves are shown in Fig. 4.

At the same time, we calculate the AUC, sensitivity, specificity, cut-off value, and Youden index. We

Table 2 The number of features grouped according to ICC

\begin{tabular}{llllll}
\hline Matrix & Poor $(I C C<0.4)$ & Fair $(0.4 \leq$ ICC $<0.6)$ & Good $(0.6 \leq I C C<0.75)$ & Excellent $(0.75 \leq I C C)$ & 206 \\
\hline GLCM & 25 & 27 & 72 & 14 & 330 \\
GLRLM & 1 & 11 & 7 & 17 & 33 \\
IH & 0 & 7 & 25 & 2 & 49 \\
NIDM & 1 & 2 & 0 & 12 & 5 \\
SHAPE & 0 & 0 & 6 & 18 \\
\hline
\end{tabular}


Table 3 Feature parameters differentiating between pulmonary inflammatory pseudotumor and peripheral lung cancer

\begin{tabular}{|c|c|c|c|c|}
\hline Category & Parent Feature & Child Feature & $P_{1}$-value & $P_{2}$-value \\
\hline \multirow[t]{22}{*}{ GLCM } & Cluster Prominence & 135-1Cluster Prominence & 0.0325 & 0.2731 \\
\hline & \multirow[t]{8}{*}{ Correlation } & 333-1 Correlation & 0.0111 & 0.0004 \\
\hline & & 333-4 Correlation & 0.0497 & 0.0070 \\
\hline & & 45-1 Correlation & 0.0128 & 0.0014 \\
\hline & & 45-4 Correlation & 0.0020 & 0.0021 \\
\hline & & 45-7 Correlation & 0.0025 & 0.0079 \\
\hline & & 90-4 Correlation & 0.0028 & 0.0029 \\
\hline & & 90-7 Correlation & 0.0086 & 0.0020 \\
\hline & & 135-7 Correlation & 0.0265 & 0.0368 \\
\hline & \multirow[t]{6}{*}{ Information Measure Corr1 } & 333-4 Information Measure Corr1 & 0.0157 & 0.1208 \\
\hline & & 0-1 Information Measure Corr1 & 0.0128 & 0.0384 \\
\hline & & 45-1 Information Measure Corr1 & $2.97 E-5$ & 0.0018 \\
\hline & & 90-1 Information Measure Corr1 & $5.7 \mathrm{E}-5$ & 0.0002 \\
\hline & & 90-4 Information Measure Corr1 & 0.0489 & 0.1070 \\
\hline & & 135-1 Information Measure Corr1 & 0.0137 & 0.0158 \\
\hline & \multirow[t]{6}{*}{ Information Measure Corr2 } & 333-1 Information Measure Corr2 & 0.0442 & 0.0140 \\
\hline & & 333-4 Information Measure Corr2 & 0.0442 & 0.0955 \\
\hline & & 0-1 Information Measure Corr2 & 0.0497 & 0.0978 \\
\hline & & 45-1 Information Measure Corr2 & 0.0020 & 0.0184 \\
\hline & & 90-1 Information Measure Corr2 & 0.0083 & 0.0130 \\
\hline & & 135-1 Information Measure Corr2 & 0.0168 & 0.0273 \\
\hline & Inverse Diff Moment Norm & 135-7 Inverse Diff moment Norm & 0.0391 & 0.2151 \\
\hline IH & Range & None & 0.0169 & 0.0232 \\
\hline NIDM & Complexity & None & 0.0268 & 0.0595 \\
\hline \multirow[t]{5}{*}{ SHAPE } & Compactness2 & None & 0.0017 & 0.0027 \\
\hline & Roundness & None & 0.0083 & 0.0102 \\
\hline & Spherical Disproportion & None & 0.0017 & 0.0020 \\
\hline & Sphericity & None & 0.0017 & 0.0028 \\
\hline & Surface Area Density & None & 0.0207 & 0.0443 \\
\hline
\end{tabular}

Note: The significant difference index of Mann-Whitney $\mathrm{U}$ test, P1-value; the significant difference index of Binary logistic regression, P2-value; indicates a significant difference $(p<0.05)$

calculated their interquartile range (IQR) and median values for features with discriminating ability, respectively. The specific values of all statistical parameters differentiating between peripheral lung cancer and PIPT are shown in Table.4.

\section{Discussion}

The results of this study indicated that there was a statistically significant difference between the radiomic features extracted from patients with peripheral lung cancer and those extracted from patients with PIPT. We assumed that this statistically significant difference between peripheral lung cancer and PIPT in this study might be related to the pathological microstructure differences between the two tissue types. Adenocarcinoma may consist of some cubic tumor cells and some columnar [32]. The pathology of primary lung adenocarcinoma is mostly papillary type, others are solid, lepidic, acinar, and micropapillary subtypes., which is often diagnosed as a mixture of multiple subtypes [33]. The pathological subtypes of lung squamous cell carcinoma include small cell, clear cell, basaloid and papillary subtypes $[6,34]$, in which papillary squamous cell carcinoma is usually characterized by exogenous endobronchial growth [35]. The histological types of small cell carcinoma are oat, intermediate and mixed subtype [6]. On pathological smears, most of the cancer cells are quasi-round or fusiform, with few cytoplasm and naked nuclei, which are very similar to lymphoma cells, and there are often mixed non-nucleated necrotic cells or extensive necrotic areas 

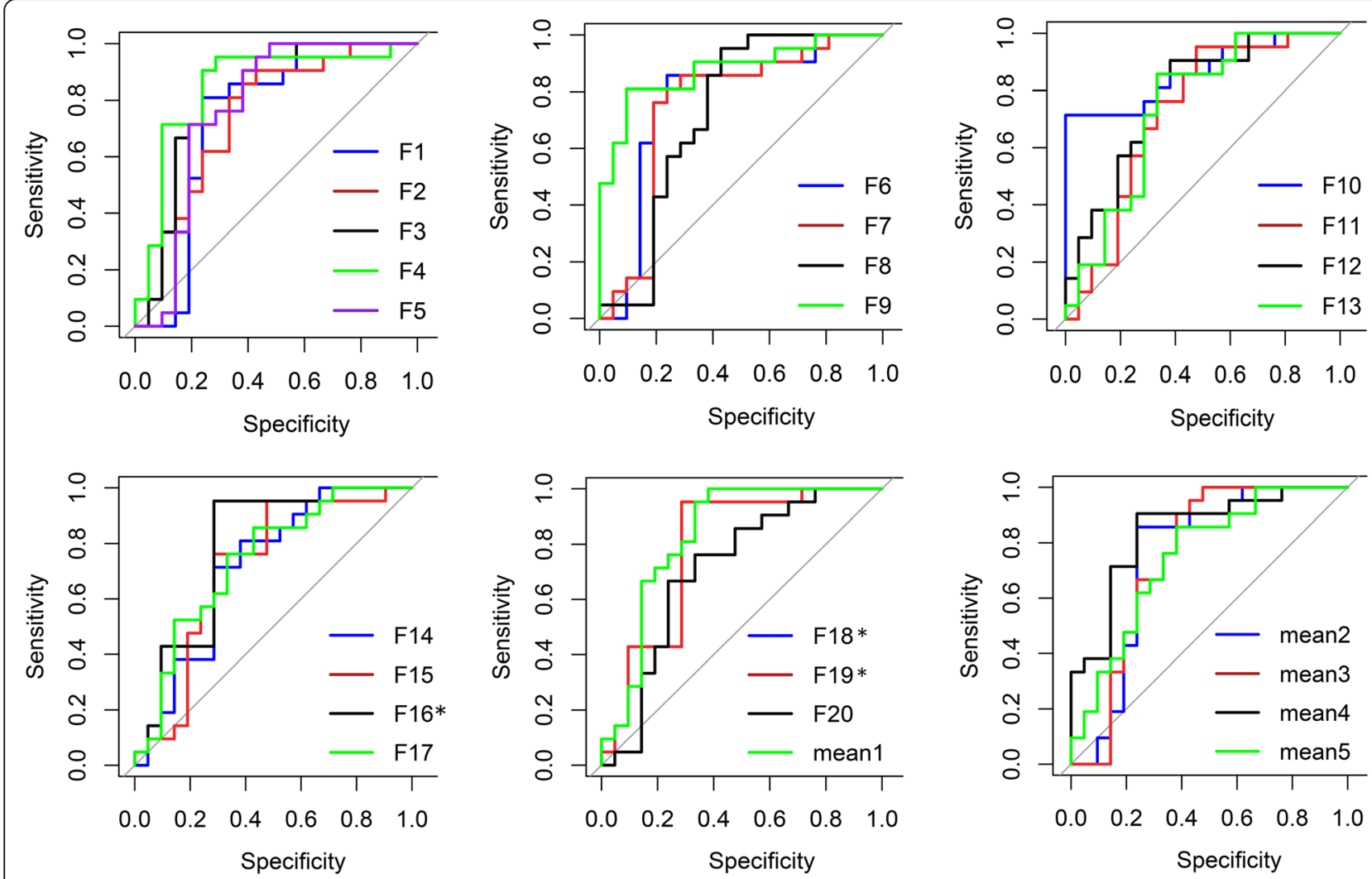

Fig. 4 ROC curses of radiomic features. * - Line coincidence

[6]. Large cell cancer cells often show sheets and nests distribution. This kind of cancer cells has rich cytoplasm, vesicular nuclei and prominent nucleoli $[6,36]$. The histological structure of PIPT is complex and often accompanied by a mixture of different numbers of cells. The main cell types of PIPT are spindle cells (myofibroblasts and fibroblasts) and chronic inflammatory cells (especially plasma cells, lymphocytes and macrophages) $[17,37,38]$. The histology of PIPT can be divided into three types: (1) fibrous histiocytic type characterized by fusiform myofibroblasts and most common (2) under the microscope, most of the patients with organized pneumonia are foam cells, eosinophils and multinucleated cells (3) Lymphocyte type is dominated by lymphocytes and plasma cells, which is the most rare type $[18,39,40]$.

The slight difference in pathology between peripheral lung cancer and pulmonary inflammatory pseudotumor may be reflected in the attenuation of CT. Correlation is a value between 0 (uncorrelated) and 1 (perfectly correlated) showing the linear dependency of gray level values to their respective voxels in the GLCM [41], Information Measure Corr1 and Information Measure Corr2 are two features that assessing the correlation between the probability distributions of two voxels (quantifying the complexity of the texture) $[41,42]$. The median values of Correlation(F1Mean3)、Information Measure Corr1(F8-Mean4) and
Information Measure Corr2(F12-Mean5) of PIPT in this study are mostly higher than that of peripheral lung cancer, which may be related to the distribution of inflammatory cells in PIPT. The specific difference of different radiomic features were described in Table 2. Relevant reports show that myofibroblasts and histiocytes play a dominant role in PIPT and are arranged in spirals or flakes $[18,39]$. This may be because PIPT necrosis is relatively less than lung cancer $[9,43,44]$, Lung cancer tissue grows rapidly and is prone to focal necrosis, which may lead to the larger values of Range(F17) and Texture Strength(F18) of PIPT compared with that of lung cancer. In this study, the shape features Compactness2, Spherical Disproportion and Spherical are the quantification of tumor roundness, and there is a certain correlation between these three features in definition. In this study, the ROC curves of these three features(F16, F18, F19) coincide, which also confirms this fact. This may be that the most of the peripheral lung cancer is spherical, and the most of the PIPT are found in the lower lobe of the lung and are round or oval $[17,20]$. Although the morphological features of peripheral lung cancer and pulmonary inflammatory pseudotumor are highly similar, the probability of occurrence of the same imaging features may be different in the cohort of patients $[39,45]$. this may be an important reason why most of the differences between 
Table 4 Statistical parameters differentiating between peripheral lung cancer and PIPT

\begin{tabular}{|c|c|c|c|c|c|c|c|c|c|c|}
\hline \multirow[t]{2}{*}{ Feature } & \multicolumn{2}{|c|}{ Peripheral Lung Cancer } & \multicolumn{2}{|l|}{ PIPT } & \multirow[t]{2}{*}{ AUC } & \multirow{2}{*}{$\begin{array}{l}\text { Confidence } \\
\text { Interval (\%) }\end{array}$} & \multirow[t]{2}{*}{ Sensitivity(\%) } & \multirow[t]{2}{*}{ Specificity(\%) } & \multirow{2}{*}{$\begin{array}{l}\text { Cutoff- } \\
\text { Value }\end{array}$} & \multirow{2}{*}{$\begin{array}{l}\text { Youden- } \\
\text { Index }\end{array}$} \\
\hline & $\mathrm{IQR}$ & median & $\mathrm{IQR}$ & median & & & & & & \\
\hline $\mathrm{F} 1$ & 0.0429 & 0.7334 & 0.0874 & 0.7913 & 0.730 & $55.74 \sim 90.29$ & 80.95 & 76.19 & 0.7657 & 0.5714 \\
\hline F2 & 0.1056 & 0.6783 & 0.1218 & 0.7846 & 0.726 & $56.02 \sim 89.11$ & 80.95 & 66.67 & 0.7401 & 0.4762 \\
\hline F3 & 0.1399 & 0.0738 & 0.1480 & 0.2719 & 0.800 & $69.04 \sim 96.94$ & 90.00 & 70.59 & 0.2047 & 0.6059 \\
\hline F4 & 0.1889 & -0.0284 & 0.2054 & 0.1434 & 0.806 & $71.76 \sim 97.85$ & 88.89 & 68.75 & 0.0246 & 0.5764 \\
\hline F5 & 0.1965 & 0.2102 & 0.1405 & 0.3946 & 0.774 & $61.64 \sim 0.9306$ & 90.48 & 65.00 & 0.3589 & 0.5548 \\
\hline F6 & 0.1370 & 0.0223 & 0.1438 & 0.1954 & 0.754 & $61.65 \sim 93.45$ & 84.21 & 72.22 & 0.1234 & 0.5643 \\
\hline F7 & 0.1467 & -0.0576 & 0.2119 & 0.410 & 0.718 & $58.29 \sim 91.37$ & 73.68 & 76.47 & -0.0316 & 0.5015 \\
\hline Mean1 & 0.1500 & 0.2280 & 0.1403 & 0.4112 & 0.800 & $77.01 \sim 98.50$ & 95.24 & 71.43 & 0.3512 & 0.6667 \\
\hline Mean2 & 0.1647 & 0.1211 & 0.1504 & 0.2990 & 0.753 & $58.75 \sim 91.82$ & 85.71 & 76.19 & 0.1855 & 0.6190 \\
\hline Mean3 & 0.1372 & 0.2362 & 0.1453 & 0.3776 & 0.751 & $58.65 \sim 91.46$ & 90.48 & 61.90 & 0.3055 & 0.5238 \\
\hline F8 & 0.0436 & -0.3821 & 0.1112 & -0.4560 & 0.726 & $55.63 \sim 89.49$ & 95.24 & 57.14 & -0.4461 & 0.5238 \\
\hline F9 & 0.0409 & -0.2944 & 0.1154 & 0.3496 & 0.878 & $77.01 \sim 98.50$ & 80.95 & 90.48 & -0.3106 & 0.7143 \\
\hline F10 & 0.0506 & -0.3605 & 0.0892 & -0.4266 & 0.864 & $75.06 \sim 97.73$ & 71.43 & 100.00 & -0.3813 & 0.7143 \\
\hline F11 & 0.0389 & -0.2849 & 0.1490 & -0.3602 & 0.723 & $55.99 \sim 88.68$ & 95.24 & 52,38 & -0.3602 & 0.4762 \\
\hline Mean4 & 0.0329 & -0.3328 & 0.0751 & -0.3978 & 0.841 & $71.78 \sim 96.48$ & 90.48 & 76.19 & -0.3624 & 0.6667 \\
\hline F12 & 0.0428 & 0.8997 & 0.0692 & 0.9302 & 0.780 & $63.75 \sim 92.26$ & 85.71 & 66.67 & 0.9175 & 0.5238 \\
\hline F13 & 0.0190 & 0.9330 & 0.0450 & 0.9623 & 0.739 & $58.18 \sim 89.66$ & 85.71 & 66.71 & 0.9567 & 0.5238 \\
\hline F14 & 0.0378 & 0.8930 & 0.0788 & 0.9202 & 0.717 & $55.49 \sim 87.82$ & 71.43 & 71.43 & 0.8975 & 0.4286 \\
\hline Mean5 & 0.0244 & 0.9065 & 0.0625 & 0.9419 & 0.751 & $60.00 \sim 90.12$ & 85.71 & 61.91 & 0.9252 & 0.4762 \\
\hline F15 & 115 & 346 & 189 & 494 & 0.717 & $54.55 \sim 88.36$ & 76.19 & 71.43 & 400.0000 & 0.4762 \\
\hline F16 & 0.1460 & 0.5928 & 0.4127 & 0.2817 & 0.785 & $63.38 \sim 93.54$ & 95.24 & 71.43 & 0.3259 & 0.6667 \\
\hline F17 & 0.1063 & 0.3569 & 0.2001 & 0.2136 & 0.739 & $58.48 \sim 89.37$ & 85.71 & 57.14 & 0.2454 & 0.4286 \\
\hline F18 & 0.0953 & 1.1904 & 0.5439 & 1.5253 & 0.785 & $63.38 \sim 93.54$ & 95.24 & 71.43 & 1.3783 & 0.6667 \\
\hline F10 & 0.0680 & 0.8400 & 0.2671 & 0.6556 & 0.785 & $63.38 \sim 93.54$ & 95.24 & 71.43 & 0.6882 & 0.6667 \\
\hline F20 & 1.7698 & 2.9145 & 5.3141 & 4.5316 & 0.710 & $54.38 \sim 87.57$ & 66.67 & 76.19 & 2.9718 & 0.4286 \\
\hline
\end{tabular}

Note: F1-GLCM-333-1-correlation, F2-CLCM-45-1-correlation, F4-GLCM-45-4-corr-elation, F6-GLCM-45-7 correlation, F6-GLCM-90-7-correlation, F7-CLCM-135-7correlation, F8-GLCM-0-1-information-measure corr1, F9-GLCM-45-1-information measure corr1, F10-GLCM-90-1-information measure corr1, F11-GLC-M-135-1information measure corr1, F12-GLCM-45-1-information measure corr2, F13-IGLCM-information measure-90-1-information corr2, F14-GLCM-135-1-information corr2, F15-IH-range, F16-shape-compactness2, F17-shape-roundness, F19-shape-sphericity, F20-shape-surface area density, mean1-mean(F2+ ... ... F4), mean2mean $(\mathrm{F} 5+\ldots \ldots \mathrm{F} 6)$, mean3-mean $(\mathrm{F} 1+\ldots \ldots \mathrm{F} 7)$, mean4-mean $(\mathrm{F} 8+\ldots \ldots \mathrm{F} 11)$, mean5-mean $(\mathrm{F} 12+\ldots \ldots+\mathrm{F} 14)$

peripheral lung cancer and PIPT in this study belong to shape features.

In addition, in this study, we found that the features extracted from different angles and distances had no special advantage in distinguishing peripheral lung cancer from PIPT. On the contrary, the features extracted from different angles and different distances have relatively high discriminating ability, such as 45-7 Correlation (F4), 45-1 Information Measure Corr1 (F9), 90-1 Information Measure Corr1 (F10). This may be related to the directionality of the microstructure of the lung in space.

Using texture features to distinguish benign and malignant tissues has been reported in many articles. Tsai et al. [46] reported that Texture features can be used to distinguish nasopharyngeal carcinoma from normal nasopharyngeal tissue, and the statistical difference in texture features between nasopharyngeal carcinoma and normal nasopharyngeal tissue may be related to the loss of stripe structure in normal nasopharyngeal tissue. and this finding had been confirmed on MRI images. Alilou et al. [47] had shown that lung adenocarcinoma and granuloma can be diagnosed by shape-based features (sphericity and roughness), and the two lesions were difficult to distinguish in pathology. These reports give great inspiration to the development of our study.

The reproducibility of radiomic features has long been an inescapable topic in the field of radiomics research. Variations in patient location, feature extraction software, imaging device and segmentation will have an unpredictable impact on the repeatability of radiomic features. If features with relatively low robustness are used in the study, they may perform poorly when tested by new data sets and even seriously affect our 
conclusions. The results of this study showed that there were differences in the repeatability of features extracted from ROI segmented by manual. As manual segmentation was easily affected by different levels of observers, we used ICC to filter features with high robustness, but this still can not meet our needs. In the follow-up research, a variety of image segmentation methods will be used to screen highly robust features and verify our conclusions, such as automatic and semi-automatic segmentation methods.

There are many limitations to our research. First of all, the number of patients is small, due to the relatively low incidence of PIPT, to a certain extent, it limits our collection of relevant medical images. In addition, this study lacks directly related pathological experimental tissue specimens, so that we can not directly confirm our findings, and the hypothesis of related problems can only be based on previous reports. Finally, all the medical images in this study are obtained from the same PET/CT model. Further studies are needed to determine whether the images obtained from different PET/CT machines can get the same conclusion. In the following research, we will enlarge the sample size as much as possible and apply machine learning and deep learning methods to verify our conclusions.

\section{Conclusions}

There were significant differences in radiological characteristics between peripheral lung cancer and PIPT, among which Information Measure Corr1 features (F11, F12) showed the highest ability to distinguish peripheral lung cancer from PIPT.

\section{Abbreviations \\ 18F-FDG PET/CT: 18F-fluorodeoxyglucose positron emission tomography/ computed tomography; PIPT: Pulmonary inflammatory pseudotumor; ROI: Regions of interest; ICC: Intra-class correlation coefficient; ROC: Receiver operating characteristic; AUC: Area under the curve; GLRLM: Gray-Level Run Length Matrix; GLCM: Gray-Level Co-occurrence Matrix; IH: Intensity Histogram; GLNIDM: Gray-level Neighbor Intensity Difference Matrix; IQR: Indicates interquartile range; FDR: False discovery rate}

\section{Acknowledgements}

Not applicable.

\section{Authors' contributions}

CLL wrote the initial draft of the manuscript. CLL and CHM contributed to conception and design, YLG and JHD, QTQ and ZHZ contributed to acquire, analyze, and interpret data. YY contributed to acquire data and enhance its intellectual content. All authors read and approved the final manuscript.

\section{Funding}

This work is supported by the National Nature Science Foundation of China (Grant Number: 81974467), Natural Science Foundation of Shandong Province (Grant Number: ZR2019MH136) and Key Research and Development Project of Shandong Province (Grant Number: 2018GSF118006). National Nature Science Foundation of China and Natural Science Foundation of Shandong Province had a role in the design of the study, collection, analysis, and interpretation of data. Key Research and Development Project of Shandong Province contributed in acquisition and writing the manuscript.

\section{Availability of data and materials}

The datasets used and analyzed during the current study available from the corresponding author on reasonable request (yinyongsd@126.com).

\section{Ethics approval and consent to participate}

The Ethics Committee (IRB) at Shandong Cancer Hospital and Institute reviewed and discussed the protocol and other aspects of this research. After a vote (Total 11, Agree 11, Disagree 0), the IRB agreed that the study followed the guidelines of good clinical practice (GCP) and that the trial could be conducted at Shandong Cancer Hospital and Institute (NO. 201905047).

\section{Consent for publication}

Not applicable.

\section{Competing interests}

The authors declare that they have no competing interests.

\section{Author details}

${ }^{1}$ School of Nuclear Science and Technology, University of South China, Hengyang 421001, China. ${ }^{2}$ Department of Radiotherapy, Shandong Cancer Hospital and Institute, Shandong First Medical University and Shandong Academy of Medical Sciences, Jinan 250117, Shandong Province, China. ${ }^{3}$ Department of Medical Imaging, Shandong Cancer Hospital and Institute, Shandong First Medical University and Shandong Academy of Medical Sciences, Jinan 250117, Shandong Province, China.

Received: 14 March 2020 Accepted: 26 June 2020

Published online: 06 July 2020

\section{References}

1. Alberg AJ, Samet JM. Epidemiology of Lung Cancer. Chest. 2003;123(1, Supplement):21S-49S.

2. Ganeshan B, Panayiotou E, Burnand K, Dizdarevic S, Miles K. Tumour heterogeneity in non-small cell lung carcinoma assessed by $C T$ texture analysis: a potential marker of survival. Eur Radiol. 2012;22(4):796-802.

3. Akata S, Yoshimura M, Nishio R, Park J, Saito K, Uchida O, Ohira T, Kato H, Okada S, Kakizaki D. High-resolution computed tomographic findings of small peripherally located squamous cell carcinoma. Clin Imaging. 2008; 32(4):259-63.

4. Stinchcombe TE, Socinski MA. Current treatments for advanced stage nonsmall cell lung Cancer. Proc Am Thorac Soc. 2009;6(2):233-41.

5. Siegel RL, Miller KD, Jemal A. Cancer statistics, 2020. CA Cancer J Clin. 2020; 70(1):7-30.

6. Travis WD. Pathology of lung Cancer. Clin Chest Med. 2011;32(4):669-92.

7. Mazzone P, Obuchowski N, Mekhail T, Meziane M, Ahmad M. Lung cancer screening: is it time for a change in policy? Cleve Clin J Med. 2007;74(6): 441-8.

8. Feng $M$, Yang $X, M a ~ Q, H e ~ Y$. Retrospective analysis for the false positive diagnosis of PET-CT scan in lung cancer patients. Medicine (Baltimore). 2017:96(42):-e7415.

9. Li CR, Li YZ, Li YM, Zheng YS. Dynamic and contrast enhanced CT imaging of lung carcinoma, pulmonary tuberculoma, and inflammatory pseudotumor. Eur Rev Med Pharmacol Sci. 2017;21(7):1588-92.

10. Way T, Chan H-P, Hadjiiski L, Sahiner B, Chughtai A, Song TK, Poopat C, Stojanovska J, Frank L, Attili A. Computer-Aided Diagnosis of Lung Nodules on CT Scans:: ROC Study of Its Effect on Radiologists' Performance. Acad Radiol. 2010;17(3):323-32.

11. Tateishi U, Nishihara H, Tsukamoto E, Morikawa T, Tamaki N, Miyasaka K. Lung tumors evaluated with FDG-PET and dynamic CT: the relationship between vascular density and glucose metabolism. J Comput Assist Tomogr. 2002;26(2):185-90.

12. Kostakoglu L, Agress H, Goldsmith SJ. Clinical role of FDG PET in evaluation of Cancer patients. RadioGraphics. 2003;23(2):315-40.

13. Chang JM, Lee HJ, Goo JM, Lee H-Y, Lee JJ, Chung J-K, Im J-G. False positive and false negative FDG-PET scans in various thoracic diseases. Korean J Radiol. 2006;7(1):57-69.

14. Rosenbaum SJ, Lind T, Antoch G, Bockisch A. False-positive FDG PET uptake -the role of PET/CT. Eur Radiol. 2006;16(5):1054-65.

15. Asad S, Aquino SL, Piyavisetpat N, Fischman AJ. False-positive FDG positron emission tomography uptake in nonmalignant chest abnormalities. Am J Roentgenol. 2004;182(4):983-9. 
16. Harada T, Watanabe K, Iwasaki A, Shirakusa T, Iwasaki H, Yosida M. Four cases of inflammatory pseudotumor. Nihon Kokyuki Gakkai Zasshi. 2003; 41(6):402-6.

17. Huellner MW, Schwizer B, Burger I, Fengels I, Schläpfer R, Bussmann C, Strobel K. Inflammatory pseudotumor of the lung with high FDG uptake. Clin Nucl Med. 2010;35(9):722-3.

18. Agrons GA, Rosado-de-Christenson ML, Kirejczyk WM, Conran RM, Stocker JT. Pulmonary inflammatory pseudotumor: radiologic features. Radiology. 1998;206(2):511-8

19. Laufer L, Cohen Z, Mares AJ, Maor E, Hirsch M. Pulmonary plasma-cell granuloma. Pediatr Radiol. 1990;20(4):289-90.

20. Sagar AES, Jimenez CA, Shannon VR. Clinical and Histopathologic Correlates and Management Strategies for Inflammatory Myofibroblastic tumor of the lung. A case series and review of the literature. Med Oncol. 2018;35(7):102.

21. Basu S, Utpat K, Joshi J. 18F-FDG PET/CT imaging features of IgG4-related pulmonary inflammatory Pseudotumor at initial diagnosis and during early treatment monitoring. J Nucl Med Technol. 2016:44(3):207-9.

22. Patnana M, Sevrukov AB, Elsayes KM, Viswanathan C, Lubner M, Menias CO. Inflammatory Pseudotumor: the great mimicker. Am J Roentgenol. 2012; 198(3):W217-27

23. Wang XL, Shan W. Application of dynamic CT to identify lung cancer, pulmonary tuberculosis, and pulmonary inflammatory pseudotumor. Eur Rev Med Pharmacol Sci. 2017;21(21):4804-9.

24. Coroller TP, Grossmann P, Hou Y, Rios Velazquez E, Leijenaar RTH, Hermann G, Lambin P, Haibe-Kains B, Mak RH, Aerts HJWL. CT-based radiomic signature predicts distant metastasis in lung adenocarcinoma. Radiother Oncol. 2015;114(3):345-50

25. Leijenaar RTH, Carvalho S, Velazquez ER, van Elmpt WJC, Parmar C, Hoekstra OS, Hoekstra CJ, Boellaard R, Dekker ALAJ, Gillies RJ, et al. Stability of FDGPET Radiomics features: an integrated analysis of test-retest and interobserver variability. Acta Oncol. 2013;52(7):1391-7.

26. Zhang L, Fried DV, Fave XJ, Hunter LA, Yang J, Court LE. IBEX: an open infrastructure software platform to facilitate collaborative work in radiomics. Med Phys. 2015;42(3):1341-53.

27. Leijenaar RTH, Carvalho S, Velazquez ER, Van Elmpt W, Parmar C, Hoekstra OS, Hoekstra CJ, Boellaard R, Dekker A, RJJAO G. Stability of FDG-PET Radiomics features: an integrated analysis of test-retest and inter-observer variability. Acta Oncol. 2013;52(7):1391-7.

28. Schmidt B, Dick A, Treutlein M, Schiller P, Bunck AC, Maintz D, Baeßler B. Intra- and inter-observer reproducibility of global and regional magnetic resonance feature tracking derived strain parameters of the left and right ventricle. Eur J Radiol. 2017:89:97-105.

29. Mann HB, Whitney DR. On a test of whether one of two random variables is stochastically larger than the other. Ann Math Stat. 1947;18(1):50-60.

30. Youden WJ. Index for rating diagnostic tests. Cancer. 1950;3(1):32-5.

31. Benjamini $Y$, Hochberg Y. Controlling the False Discovery Rate: a Practical and Powerful Approach to Multiple Testing. J R Stat Soc B. 1995;57(1):289-300.

32. Edwards A. Tumours of the lung. Br J Surg. 2005;26:166-92.

33. Casteillo F, Guy J-B, Dal-Col P, Karpathiou G, Pommier B, Bayle-Bleuez S, Fournel $P$, Vassal F, Forest F. Pathologic subtypes of lung adenocarcinoma brain metastasis is a strong predictor of survival after resection. Am J Surg Pathol. 2018;42(12):1701-7.

34. Churg A, Johnston WH, Stulbarg M. Small cell squamous and mixed small cell squamous--small cell anaplastic carcinomas of the lung. Am J Surg Pathol. 1980;4(3):255-63.

35. Dulmet-Brender E, Jaubert F, Huchon G. Exophytic endobronchial epidermoid carcinoma. Cancer. 1986;57(7):1358-64.

36. Travis WD, Brambilla E, Müller-Hermelink HK, Harris CC. Pathology and genetics of tumours of the lung, pleura, thymus and heart (WHO classification of tumours). Lyon: IARC Press; 2004.

37. Berardi RS, Lee SS, Chen HP, Stines GJ. INFLAMMATORY PSEUDOTUMORS OF THE LUNG; 1983.

38. Matsubara O, Tan-Liu NS, Kenney RM, Mark EJ. Inflammatory pseudotumors of the lung: progression from organizing pneumonia to fibrous histiocytoma or to plasma cell granuloma in 32 cases. Hum Pathol. 1988; 19(7):807-14.

39. Schlimper C, Sommer T, Flacke S, Wolff M, Schild H, Kreft B. Radiologische Befunde bei inflammatorischen Pseudotumoren. In: Rontg R-F, editor. Stuttgart: 2005: @) Georg Thieme Verlag KG; 2005. p. 1506-12.

40. Coffin CM, Watterson J, Priest JR, Dehner LP. Extrapulmonary inflammatory myofibroblastic tumor (inflammatory pseudotumor). A clinicopathologic and immunohistochemical study of 84 cases. Am J Surg Pathol. 1995;19(8): 859-72.

41. Haralick RM, Shanmugam K, Dinstein $\mathbb{I H}$. Textural features for image classification. IEEE Transactions on systems, man, and cybernetics. IEEE Trans Syst Man Cybern. 1973;3(6):610-21.

42. Linfoot EH. An informational measure of correlation. Inf Control. 1957;1(1): 85-9.

43. Zennaro H, Laurent F, Vergier B, Rauturier J, Airaud J, Jougon J, Drouillard J. Inflammatory myofibroblastic tumor of the lung (inflammatory pseudotumor): uncommon cause of solitary pulmonary nodule. Eur Radiol. 1999;9(6):1205-7.

44. Hammas N, Chbani L, Rami M, Boubbou M, Benmiloud S, Bouabdellah Y, Tizniti S, Hida M, Amarti A. A rare tumor of the lung: inflammatory myofibroblastic tumor. Diagn Pathol. 2012;7(1):83.

45. Ebara K, Takashima S, Jiang B, Numasaki H, Fujino M, Tomita Y, Nakanishi K, Higashiyama M. Pleural invasion by peripheral lung cancer: prediction with three-dimensional CT. Acad Radiol. 2015;22(3):310-9.

46. Tsai A, Buch K, Fujita A, Qureshi MM, Kuno H, Chapman MN, Li B, Oda M, Truong MT, Sakai O. Using CT texture analysis to differentiate between nasopharyngeal carcinoma and age-matched adenoid controls. Eur J Radiol. 2018;108:208-14.

47. Alilou M, Beig N, Orooji M, Rajiah P, Velcheti V, Rakshit S, Reddy N, Yang M, Jacono F, Gilkeson RC. An integrated segmentation and shape-based classification scheme for distinguishing adenocarcinomas from granulomas on lung CT. Med Phys. 2017;44(7):3556-69.

\section{Publisher's Note}

Springer Nature remains neutral with regard to jurisdictional claims in published maps and institutional affiliations.
Ready to submit your research? Choose BMC and benefit from:

- fast, convenient online submission

- thorough peer review by experienced researchers in your field

- rapid publication on acceptance

- support for research data, including large and complex data types

- gold Open Access which fosters wider collaboration and increased citations

- maximum visibility for your research: over $100 \mathrm{M}$ website views per year

At BMC, research is always in progress.

Learn more biomedcentral.com/submissions 